\title{
Channel Reservation in Cognitive Radio Networks with the RESTART Retransmission Strategy
}

\author{
S. Lirio Castellanos-Lopez, Felipe A. Cruz-Pérez \\ Elect. Eng. Dept., CINVESTAV-IPN \\ Mexico City, Mexico \\ lirio@cinvestav.mx, facruz@cinvestav.mx
}

\author{
Genaro Hernandez-Valdez \\ Electronics Department, UAM-A \\ Mexico City, Mexico \\ ghv@correo.azc.uam.mx
}

\begin{abstract}
In this paper, channel reservation to prioritize ongoing secondary users' (SUs) calls over new SUs' requests in cognitive radio networks (CRNs) with delay tolerant traffic and the RESTART (also known as preemptive repeat identical) retransmission strategy is analyzed and evaluated. Additionally, an analytical approach to approximately evaluate the performance of CRNs under the RESTART retransmission strategy is proposed and developed. It is shown that channel reservation is an effective mechanism both to reduce the negative impact of retransmissions and to widen the conditions under which system stability can be achieved. To the best of the authors' knowledge, mechanisms to modify the failure process in CRNs have been neither analyzed nor evaluated in the literature.
\end{abstract}

Keywords: Cognitive radio networks; RESTART retransmission strategy; teletraffic and performance analysis; delay tolerant traffic.

\section{INTRODUCTION}

Cognitive radio networks (CRNs) have been proposed to both enhancing spectrum usage and solving the problem of heterogeneity of radio devices [1]. A CRN consists of two types of wireless networks operating in a given common service area. The one that owns the license for spectrum usage is referred to as the primary system, and the calls generated by users of this network (primary users) constitute the primary traffic (PT) stream. The other network in the same service area is referred to as the secondary system, which opportunistically shares the spectrum resource with the primary system. The calls generated by users of the secondary system constitute the secondary traffic (ST) stream. The primary users (PUs) always have a higher channel access priority over secondary users (SUs). When an idle primary channel is detected, SUs may temporally occupy this unused channel. If a PU decides to access the primary channel, all SUs using this channel must relinquish their transmission immediately. These unfinished cognitive calls may be either simply blocked [1]-[4] or switched to an idle channel, if one is available, to continue the call (this process is called spectrum handoff). Moreover, to reduce the impact of service interruption, for delay tolerant services, if no vacant channels are available, interrupted SUs may be queued in a buffer to wait for the releasing of an occupied channel [5]-[8]. In this case, when a queued SU finds a new available channel, it is allowed to continue transmitting its information. In this work, two simple retransmission strategies to handle with queued SUs in CRNs are considered: RESTART and Resume (RR) [12]. In the RESTART strategy ${ }^{1}$,

\footnotetext{
${ }^{1}$ The RESTART strategy is relevant, for example, in applications that employ the FTP and HTTP protocols, or in receiving costumer service by telephone, among others [9].
}

when an ongoing secondary session is interrupted, all the information transmitted (i.e., packets, frames, or other data units) until the interruption point is lost and the SU must start over again when allowed to occupy an available primary channel [9], [12]. On the other hand, in the RR strategy, when an ongoing secondary session is interrupted, it knows where it exactly stops and can resume at that point when it leaves the queue to be reconnected to the system [5]-[8] .

Authors in [9] state that the RESTART strategy has resisted detailed analysis. In [10]-[11] it is shown that the Laplace transform of the total time to complete the job (total time a task spends executing because of the failures, but not including the time it spends waiting) under the RESTART strategy was not matrix exponential, and therefore it cannot be solved in the Markov chain framework. In this research direction, authors in [12]-[13] show that, if the distribution of the unencumbered service time (i.e., total time a task spends executing without failures) has an exponential tail, the total time to complete the job is power tailed. In a related work [14]-[15], it has been shown that, under the assumption that the packet size distribution has infinite support, all retransmission-based protocols could cause heavy-tailed behavior and possibly even zero throughput. These heavy tails can result entirely from retransmissions, even when the service time and channel characteristics are light-tailed. Authors in [16] realize that, in practice, packet sizes are bounded by the maximum transmission unit and this motivate them to investigate the impact of retransmission on network performance by allowing the packet sizes to have finite support.

In a related work [19]-[20], we compare the performance between the RESTART and RR strategies; however, no mechanism to reduce the negative impact of retransmission is employed. In [19]-[20], the system performance under the RESTART strategy was only evaluated by simulation. In [19], the service time distribution of interrupted SUs under the RS strategy was derived. Additionally, in [19], for the RR strategy, a trellis-based mathematical analysis is developed to compute the secondary users' mean total transmission delay.

To the best of our knowledge, mechanisms used to modify the failure process (i.e., for reducing failure rate) have been neither analyzed nor evaluated in the literature ${ }^{3}$. Moreover, the performance of CRNs under the RESTART retransmission

\footnotetext{
${ }^{2}$ The RR retransmission strategy can be directly implemented when automatic repeat request-based error control protocols (i.e., hybrid ARQ, selective-reject ARQ) are used as the receiver must acknowledge received data.

${ }^{3}$ In [16], power control at the physical layer is mentioned as a way to change the relationship between the channel dynamics and the units in which packets should be transmitted in order to achieve the best network performance. However, its performance it is neither analyzed nor evaluated.
} 
strategy has been not mathematically analyzed in the open literature. In this paper, channel reservation to prioritize ongoing over new-secondary-session requests in CRNs is proposed and it is shown that it is an effective mechanism both to reduce the negative impact of retransmissions and to widen the conditions under which system stability can be achieved. Our contribution includes the development of an analytical approach to approximately evaluate the performance for CRNs under the RESTART retransmission strategy. Note that channel reservation was considered in [1] and [4] to prioritize ongoing over new secondary session requests in CRNs but in a Blocked Calls Cleared (BCC) model. Contrary to [1] and [4], in this paper a Blocked Calls Delayed (BCD) model is considered.

\section{SYSTEM MODEL}

The CRN model used in [5]-[7] is adopted here. It is assumed that there are $M$ channels. To reduce the interruption of ST calls upon the arrival of PT calls, a number $r$ of channels is precluded to be used by ST calls (notice that these channels can be considered as reserved for exclusive use of PT calls). If there are $r$ or less idle channels and a secondary-session arrives, it is blocked and considered lost from the system. When an ongoing SU detects or is informed of an arrival of PT call in its current channel, it immediately leaves the channel and switches to an idle channel, if one is available, to continue the call. If at that time all the channels are occupied, the ST call is placed into a buffer located at its BS. The queued ST calls are served in first-come first-served order. It is assumed that ST calls can wait indefinitely to be served. Additionally, for mathematical tractability, it is assumed that there exists no limit in the number of reconnections that an ongoing ST call can perform. In this manner, the maximum number of queued ST calls is $M-r$, which corresponds to the limiting case that all the $M-r$ ongoing calls are ST calls and are eventually preempted to the queue due to the arrivals of PT calls. Thus, a finite queue of length $M-r$ is considered.

Arrivals of the PT and ST calls are assumed to form independent Poisson processes with rates $\lambda^{(P)}$ and $\lambda^{(S)}$, respectively. The random variable (RV) used to represent the PUs' inter-arrival time is $\mathbf{X}_{a}$. Service time for PUs is considered exponentially distributed with rate $\mu^{(P)}$. The RV used to represent this time variable is $\mathbf{X}_{S}{ }^{(P)}$. The corresponding unencumbered service time (that is, the time a SU uses a channel without interruptions) for SUs is modeled either as a negative exponential, 2nd order Coxian, or uniform distributed $\mathrm{RV}$. The RV used to represent this time is $\mathbf{X}_{S}{ }^{(S)}$. Fig. 1 shows a diagram of phases of a $n$-th order Coxian distribution. Notice that $\beta_{i}$ (for $i=1,2, \ldots, n-1$ ) represents the probability that the absorbing state is reached after the $i$-th phase. For a 2 nd order Coxian distribution, $\beta_{2}=1$ and $\beta_{1}=\beta$. The $i$-th phase of this distribution is an independent exponential RV with parameter $\mu_{i}^{(S)}$ for $(i=1,2)$. The RVs used to represent these times are $\mathbf{X}_{i}^{(S)}$ (for $\left.i=1,2\right)$. The mean service time for SUs is denoted by $1 / \mu^{(S)}$.

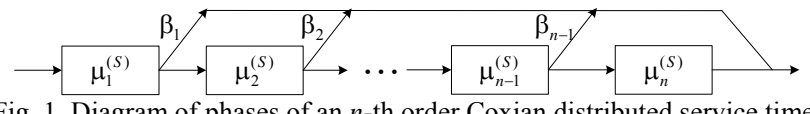

Fig. 1. Diagram of phases of an $n$-th order Coxian distributed service time.

\section{TELETRAFFIC ANALYSIS UNDER THE RR STRATEGY}

In this section, the teletraffic analysis for the performance evaluation of CRNs under the RR strategy is developed. Service time for SUs is assumed two-phase Coxian distributed. In the RR strategy, when a queued $\mathrm{SU}$ is reconnected to the system, it transmits its information (i.e., packets, frames, or data units) starting at the point it was preempted. As the permanence time in the different phases of service time is exponentially distributed, it is sufficient to know the phase where the interruption occurred due to the Markovian property. Thus, it is possible to keep track in a single state variable the number of users in each phase of the service time for both ongoing and queued SUs. Then, a four-dimensional birth and death process is required for modeling this system. Each state variable is denoted by $k_{i}$ (for $i=0,1,2,3$ ). $k_{0}$ represents the number of ongoing PUs, $k_{1}\left(k_{2}\right)$ the number of ongoing SUs in phase 1 (2), and $k_{3}$ the number of SUs in the queue that were interrupted in phase 1. Let us define the following vectors: $\mathbf{k}=\left(k_{0}, k_{1}, k_{2}, k_{3}\right)$, and $\mathbf{e}_{i}$ is a unit vector of four elements, whose entries are 0 except the $i$-th position which is 1 (for $i=0, \ldots, 3$ ). Considered the state $\mathbf{k}$ as the reference one, a detailed description of all the possible transition rates from and toward state $\mathbf{k}$ are given as follows. The call birth rate for PUs or SUs generating a transition from state $\mathbf{k}$ to state $\mathbf{k}+\mathbf{e}_{i}$ is given by

$$
a_{i}(\mathbf{k})= \begin{cases}\lambda^{(P)} & \sum_{j=0}^{2} k_{j}<M ; k_{0} \geq 0 ; k_{3}=0 ; i=0 \\ \lambda^{(S)} & \sum_{j=0}^{2} k_{j}<M-r ; k_{1} \geq 0 ; k_{3}=0 ; i=1 \\ 0 & ; \text { otherwise }\end{cases}
$$

The second rate is due to the fact that $r$ channels are reserved for exclusive use of PT calls. The call death rate for PUs or SUs generating a transition from $\mathbf{k}$ to $\mathbf{k}-\mathbf{e}_{i}$ is given by

$$
b_{i}(\mathbf{k})= \begin{cases}k_{0} \mu_{s}^{(P)} \quad \sum_{i=0}^{2} k_{i} \leq M ; k_{3}=0 ; i=0 \\ \beta k_{1} \mu_{1}^{(S)} \quad \sum_{i=0}^{2} k_{i} \leq M ; \sum_{i=1}^{2} k_{i} \leq M-r ; k_{3}=0 ; i=1 \\ k_{2} \mu_{2}^{(S)} \quad \sum_{i=0}^{2} k_{i} \leq M ; \sum_{i=1}^{2} k_{i} \leq M-r ; k_{3}=0 ; i=2 \\ 0 \quad ; \text { otherwise }\end{cases}
$$

The transition rate of the service time of a SU from phase 1 to 2 generating a transition from $\mathbf{k}$ to $\mathbf{k}-\mathbf{e}_{\mathbf{1}}+\mathbf{e}_{\mathbf{2}}$ is given by

$$
c(\mathbf{k})=\left\{\begin{array}{l}
(1-\beta) k_{1} \mu_{1}^{(S)} ; \sum_{i=0}^{3} k_{i} \leq 2 M-r ; \sum_{i=1}^{3} k_{i} \leq M-r ; k_{2} \geq 0 \\
0 \quad ; \text { otherwise }
\end{array}\right.
$$

The transition rate of queuing a SU in phase 1 (due to the arrival of a PU at the first stage of its service time) generating a transition from $\mathbf{k}$ to $\mathbf{k}+\mathbf{e}_{0}-\mathbf{e}_{1}+\mathbf{e}_{3}$ is given by

$$
d_{1}(\mathbf{k})=\left\{\begin{array}{cc}
\frac{k_{1}}{M-k_{0}} \lambda^{(P)} & ; 0 \leq k_{0}<M ; \sum_{j=0}^{2} k_{j} \geq M ; \sum_{j=0}^{3} k_{j}<2 M-r ; \\
& ; k_{3} \geq 0 ; \sum_{i=1}^{3} k_{i} \leq M-r \\
0 \quad \text { otherwise } &
\end{array}\right.
$$

The transition rate of queuing a $\mathrm{SU}$ in phase 2 (due to the arrival of a PU at the second stage of its service time) generating a transition from $\mathbf{k}$ to $\mathbf{k}+\mathbf{e}_{0}$ is given by 


$$
d_{2}(\mathbf{k})= \begin{cases}\frac{M-k_{0}-k_{1}}{M-k_{0}} \lambda^{(P)} & ; 0 \leq k_{0}<M ; \sum_{j=0}^{2} k_{j} \geq M ; \sum_{j=0}^{3} k_{j}<2 M-r ; \\ & ; \sum_{i=1}^{3} k_{i} \leq M-r \\ 0 \quad \text {;otherwise } & \end{cases}
$$

The reconnection rate of a $\mathrm{SU}$ in phase 1 due to the death of a PU generating a transition from $\mathbf{k}$ to $\mathbf{k}-\mathbf{e}_{0}+\mathbf{e}_{1}-\mathbf{e}_{3}$ is

$$
e_{0}(\mathbf{k})= \begin{cases}\frac{k_{3}}{k_{3}+\sum_{j=0}^{2} k_{j}-M} k_{0} \mu^{(P)} & ; k_{0} \leq M ; \sum_{j=0}^{2} k_{j} \geq M ; \sum_{j=0}^{3} k_{j} \leq 2 M-r ; \\ 0 \quad ; \text { otherwise } & ; k_{1} \geq 0 ; k_{3}>0 ; \sum_{j=1}^{3} k_{j} \leq M-r ; i=0\end{cases}
$$

The reconnection rate of a SU in phase 2 due to the death of a PU generating a transition from $\mathbf{k}$ to $\mathbf{k}-\mathbf{e}_{0}$ is given by

$$
e_{1}(\mathbf{k})= \begin{cases}\frac{\sum_{j=0}^{2} k_{j}-M}{k_{3}+\sum_{j=0}^{2} k_{j}-M} k_{0} \mu^{(P)} ; k_{0} \leq M ; \sum_{j=0}^{2} k_{j}>M ; \sum_{j=0}^{3} k_{j} \leq 2 M-r ; \\ 0 \quad ; \sum_{j=1}^{3} k_{j} \leq M-r ; i=1\end{cases}
$$

The reconnection rate of a $\mathrm{SU}$ in phase 1 due to the death of a SU in phase 1 generating a transition from $\mathbf{k}$ to $\mathbf{k}-\mathbf{e}_{3}$ is

$$
e_{2}(\mathbf{k})= \begin{cases}\frac{k_{3}}{k_{3}+\sum_{j=0}^{2} k_{j}-M} \beta k_{1} \mu_{1}^{(S)} & ; \sum_{j=0}^{2} k_{j} \geq M ; \sum_{j=0}^{3} k_{j} \leq 2 M-r ; \\ 0 \quad ; \text { otherwise } & ; k_{3}>0 ; \sum_{j=1}^{3} k_{j} \leq M-r ; i=2\end{cases}
$$

The reconnection rate of a SU in phase 2 due to the death of a $\mathrm{SU}$ in phase 1 generating a transition from $\mathbf{k}$ to $\mathbf{k}-\mathbf{e}_{1}$ is

$$
e_{3}(\mathbf{k})= \begin{cases}\frac{\sum_{j=0}^{2} k_{j}-M}{k_{3}+\sum_{j=0}^{2} k_{j}-M} \beta k_{1} \mu_{1}^{(S)} & ; \sum_{j=0}^{2} k_{j}>M ; \sum_{j=0}^{3} k_{j} \leq 2 M-r ; \\ & ; \sum_{j=1}^{3} k_{j} \leq M-r ; i=3\end{cases}
$$

The reconnection rate of a $\mathrm{SU}$ in phase 1 due to the death of a SU in phase 2 generating a transition from $\mathbf{k}$ to $\mathbf{k}-\mathbf{e}_{2}+\mathbf{e}_{1}-\mathbf{e}_{3}$ is

$$
e_{4}(\mathbf{k})=\left\{\begin{array}{l}
\frac{k_{3}}{k_{3}+\sum_{j=0}^{2} k_{j}-M}\left(M-k_{0}-k_{1}\right) \mu_{2}^{(S)} \quad ; \sum_{j=0}^{2} k_{j} \geq M ; \sum_{j=0}^{3} k_{j} \leq 2 M-r \\
\quad ; k_{1} \geq 0 ; k_{3}>0 ; \sum_{j=1}^{3} k_{j} \leq M-r ; i=4 \\
0 \text {;otherwise }
\end{array}\right.
$$

The reconnection rate of a SU in phase 2 due to the death of a SU in phase 2 generating a transition from $\mathbf{k}$ to $\mathbf{k}-\mathbf{e}_{2}$ is

$$
e_{5}(\mathbf{k})= \begin{cases}\frac{\sum_{j=0}^{2} k_{j}-M}{k_{3}+\sum_{j=0}^{2} k_{j}-M}\left(M-k_{0}-k_{1}\right) \mu_{2}^{(S)} & ; \sum_{j=0}^{2} k_{j}>M ; \sum_{j=0}^{3} k_{j} \leq 2 M-r ; \\ & ; \sum_{j=1}^{3} k_{j} \leq M-r ; i=5 \\ 0 \quad \text { otherwise } & \end{cases}
$$

The valid space state is given by $\Omega_{0}=\left\{\mathbf{k} \mid k_{i} \geq 0, \sum_{i=0}^{3} k_{i} \leq 2 M, \sum_{i=1}^{3} k_{i} \leq M, k_{3}=0\right.$ if $\left.\sum_{i=0}^{2} k_{i}<M ; \quad i=0,1,2,3\right\}$

The steady state probabilities balance equation is given by $\left[\sum_{i=0}^{1} a_{i}(\mathbf{k})+\sum_{i=0}^{2} b_{i}(\mathbf{k})+c(\mathbf{k})+\sum_{i=1}^{2} d_{i}(\mathbf{k})+\sum_{i=0}^{5} e_{i}(\mathbf{k})\right] P(\mathbf{k})=$ $\sum_{i=0}^{1} a_{i}\left(\mathbf{k}-\mathbf{e}_{i}\right) P\left(\mathbf{k}-\mathbf{e}_{i}\right)+\sum_{i=0}^{2} b_{i}\left(\mathbf{k}+\mathbf{e}_{i}\right) P\left(\mathbf{k}+\mathbf{e}_{i}\right)+c\left(\mathbf{k}+\mathbf{e}_{1}-\mathbf{e}_{2}\right) P\left(\mathbf{k}+\mathbf{e}_{1}-\mathbf{e}_{2}\right)+$ $d_{1}\left(\mathbf{k}-\mathbf{e}_{0}+\mathbf{e}_{1}-\mathbf{e}_{3}\right) P\left(\mathbf{k}-\mathbf{e}_{0}+\mathbf{e}_{1}-\mathbf{e}_{3}\right)+d_{2}\left(\mathbf{k}-\mathbf{e}_{0}\right) P\left(\mathbf{k}-\mathbf{e}_{0}\right)+$ $e_{0}\left(\mathbf{k}+\mathbf{e}_{0}-\mathbf{e}_{1}+\mathbf{e}_{3}\right) P\left(\mathbf{k}+\mathbf{e}_{0}-\mathbf{e}_{1}+\mathbf{e}_{3}\right)+e_{1}\left(\mathbf{k}+\mathbf{e}_{0}\right) P\left(\mathbf{k}+\mathbf{e}_{0}\right)+$ $e_{2}\left(\mathbf{k}+\mathbf{e}_{3}\right) P\left(\mathbf{k}+\mathbf{e}_{3}\right)+e_{3}\left(\mathbf{k}+\mathbf{e}_{1}\right) P\left(\mathbf{k}+\mathbf{e}_{1}\right)+$ $e_{4}\left(\mathbf{k}+\mathbf{e}_{2}-\mathbf{e}_{1}+\mathbf{e}_{3}\right) P\left(\mathbf{k}+\mathbf{e}_{2}-\mathbf{e}_{1}+\mathbf{e}_{3}\right)+e_{5}\left(\mathbf{k}+\mathbf{e}_{2}\right) P\left(\mathbf{k}+\mathbf{e}_{2}\right)$

An arrival of a new cognitive call is blocked when there is not idle channel. That is, new call blocking probability $P_{B}{ }^{(\mathrm{S})}$ can be computed as follows

$$
P_{B}^{(S)}=\sum_{k_{0}=0}^{M} \sum_{k_{1}=0}^{\max \left(0, M-r-k_{0}\right) \max \left(0, M-r-k_{0}\right)-k_{1}} \sum_{k_{2}=0} P(\mathbf{k})
$$

On the other hand, the probability that an SU be interrupted upon the arrival of a PU can be computed as follows [2]

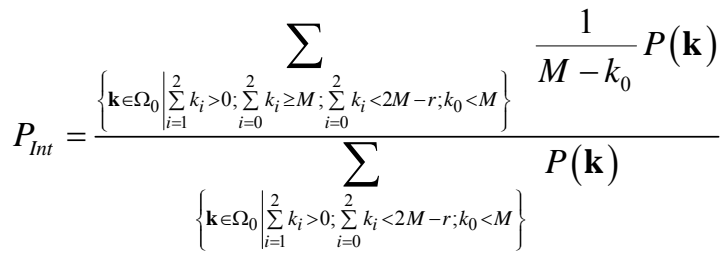

\section{AnAlytical APPROACH BASED ON THE APPROXIMATON OF THE DISTRIBUTION OF THE TOTAL TRANSMISSION TIME}

In this section, we proposed a novel and simple approximated methodology to analyze (under a Markov chain framework) CRNs operating under the RESTART retransmission strategy. To this aim, the RESTART strategy is evaluated using the queuing model of the RR strategy considering the total transmission time in RESTART as the unencumbered service time of SUs. Fig. 2 shows that a SU call with unencumbered service time $x_{s}^{(S)}$ has a total transmission time $x$, which consists of a number of preempted (interrupted) secondary service times $x_{i}$ and one successful unencumbered service time $x_{s}^{(S)}$. Also, Fig. 2 shows that if $x$ is considered as the unencumbered service time in the RR strategy, then total channel occupancy is the same in both RESTART and Resume strategies. The problem is to determine the pdf of the total transmission time in the RESTART strategy and approximate it by a suitable distribution. As the pdf of the total transmission time depends on the failure rate (i.e., call arrival rate of PUs, $\lambda^{(P)}$, and the interruption probability upon the arrival of primary users, $\left.P_{\text {Int }}\right)$ and vice versa, it is necessary to employ a fix point iteration method. The approximated mathematical analysis approach works as follows.

Step 0: Set $P_{\text {Int }}=0$ and assume a pdf of the unencumbered service time for SUs. Go to Step 1. 
Step 1: Calculate the moments of the total transmission time of SUs considering the actual value of $P_{\text {Int }}$. For this calculation, the expressions obtained in [12]-[13] are used, considering that the failure rate is $P_{\text {Int }} \lambda^{(P)}$ and the predefined pdf of the unencumbered service time for SUs. Go to Step 2.

Step 2: Approximate the pdf of the total transmission time of SUs by means of a phase-type pdf (in the numerical results section, negative exponential and order 2 Coxian distributions are used) with the moments calculated in Step 1. Go to Step 3.

Step 3: The RESTART strategy is analyzed using the teletraffic model developed in Section $\mathrm{V}$ to analyze the resume retransmission strategy. The key aspect is to use in that queuing model the pdf of the total transmission time of SUs instead of the secondary unencumbered service time. Go to Step 4.

Step 4: Calculate $P_{\text {Int }}$ (i.e., for the case when a $2^{\text {nd }}$ order Coxian is considered to approximate the pdf of the total transmission time, it is given by eq. (2)). If the just calculated value of $P_{\text {Int }}$ differs (for more than a predefined value) from its previous considered value, then go to Step 1 considering the calculated value of $P_{\text {Int }}$. Otherwise, go to Step 5.

Step 5: Obtain performance metrics and end.

The accuracy of our proposed methodology is validated by extensive set of simulation results and different distributions of the unencumbered service time of SUs. It is observed that the proposed analytical approach works for low to moderate values of the failure rate. For high values of the failure rate, the number of retransmissions increases and this fact impacts on the heavy-tailed characteristic of the total transmission time distribution and as consequence some relevant moments of this distribution become infinite. Thus, the considered phase-type distribution cannot capture this behavior.

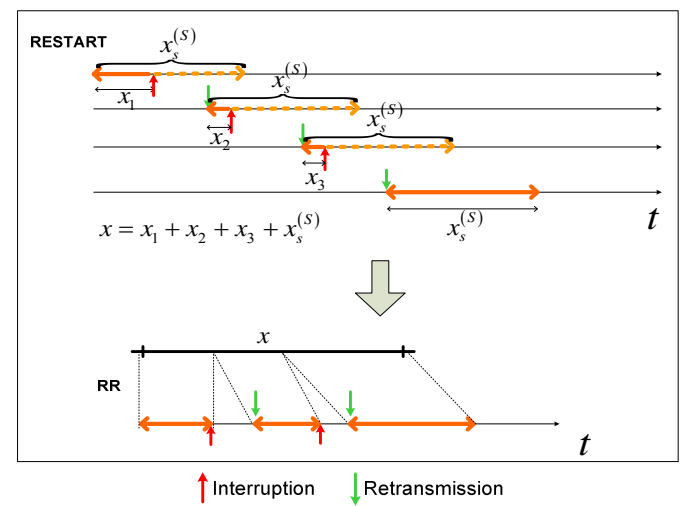

Fig. 2. Equivalence of the CHT in the RESTART strategy with unencumbered service time $x_{s}^{(S)}$ and the RR strategy with unencumbered service time equal to the total transmission time $x$ in RESTART.

\section{NuMERICAL RESUltS}

The goal of the numerical evaluations is both to validate our proposed analytical models and quantify the benefits of channel reservation to reduce the negative impact of retransmissions. Different distributions of the service time of secondary sessions are presented: negative-exponential, 2-nd order Coxian, and uniform. System performance is evaluated in terms of SU new call blocking probability, $P_{I n t}$, and SU mean normalized transmission delay. The mean normalized transmission delay is the difference between the mean value of the elapsed time from the epoch the SU arrives to the system to the epoch it finally leaves the system and the mean service time, normalized to the mean service time. Unless otherwise specified, the following values of the system parameters are used: $M=3,1 / \mu^{(P)}=16.7 \mathrm{~s}$, and $1 / \mu^{(S)}=1.22 \mathrm{~s}$. Similar values of the these parameters were considered in [1], [2] and [4]. Table I shows other system parameters used here.

TABLE I. SYSTEM PARAMETERS FOR THE DIFFERENT SERVICE TIME DISTRIBUTIONS CONSIDERED IN THIS SECTION.

\begin{tabular}{|l|r|r|r|r|r|}
\hline Service time distribution & \multicolumn{1}{|l|}{$\mu_{1}^{(S)}$} & \multicolumn{1}{l|}{$\mu_{2}^{(S)}$} & \multicolumn{1}{l|}{$\boldsymbol{l}$} & \multicolumn{1}{l|}{ SV } \\
\hline EXP-NEG & 0.82 & N/A & 1 & 1 & 2 \\
\hline COXIAN order 2 & 8.1842 & 0.018 & 0.9801 & 9 & 15 \\
\hline
\end{tabular}

Figs. 3 and 4 (5 and 6) show, respectively, SU new call blocking probability and SU normalized transmission delay as function of both primary and secondary mean arrival rates for the case when service time for SU is exponentially distributed (2-nd order Coxian distributed) and the pdf of the total transmission time is approximated with the exponential $\left(2^{\text {nd }}\right.$ order Coxian) distribution. On the other hand, Fig. 7 shows SU new call blocking probability as function of both primary and secondary mean arrival rates for the case when service time for $\mathrm{SU}$ is uniform distributed and the total transmission time is approximated with an exponential negative distribution. From Figs. 3, 5, and 7, perfect agreement is observed between analytical and simulation results, which validates our proposed analytical model. The main conclusion that can be extracted from Figs. 3 and 7 is that, from the blocking probability point of view and assuming that the service time of SU follows either an exponential or a uniform distribution, the performance of RR and RESTART strategies is very similar irrespective of the values of both primary and secondary mean arrival rates. On the other hand, Fig. 4 shows that, from the point of view of the transmission delay, the RR strategy slightly outperforms the behavior of the RESTART strategy. Specifically, Fig. 4 shows that the difference in performance between these two strategies increases as the mean arrival rate of PUs increases. The reason of this behavior is evident: Contrary to the RESTART strategy, in the RR strategy an interrupted SU does not need to transmit again the information bits transmitted before its previous connection was interrupted. This fact contributes to improve delay transmission. Figs. 5 and 6 show that, for high traffic load of PUs, the RR strategy significantly outperforms the RESTART strategy when the service time of SU is modeled as a 2-nd order Coxian distributed RV. This behavior becomes more evident as the mean arrival rate of PUs increases. The reason of this behavior is due to the bias effect on the service time distribution of preempted SUs explained in Section III. From Fig. 5, it is important to note that some analytical results for the RESTART retransmission strategy could not be obtained. This is due to the fact that as the traffic load of both PUs and SUs increase, the retransmissions (and as consequence the failure rate) increases. This fact impacts on the heavy-tailed characteristic of the total transmission time distribution and as consequence some relevant moments of this distribution become infinite. Also Fig. 5 reveals that the accuracy of the analytical results is irrelevant to the type of distribution (exponential or $2^{\text {nd }}$ order Coxian) that is used to approximate the total transmission time of SUs. Then, for simplicity the exponential approximation may be preferred. 




Fig. 3. Blocking probability with exponentially distributed service time and the total transmission time approximated by an exponential distribution.

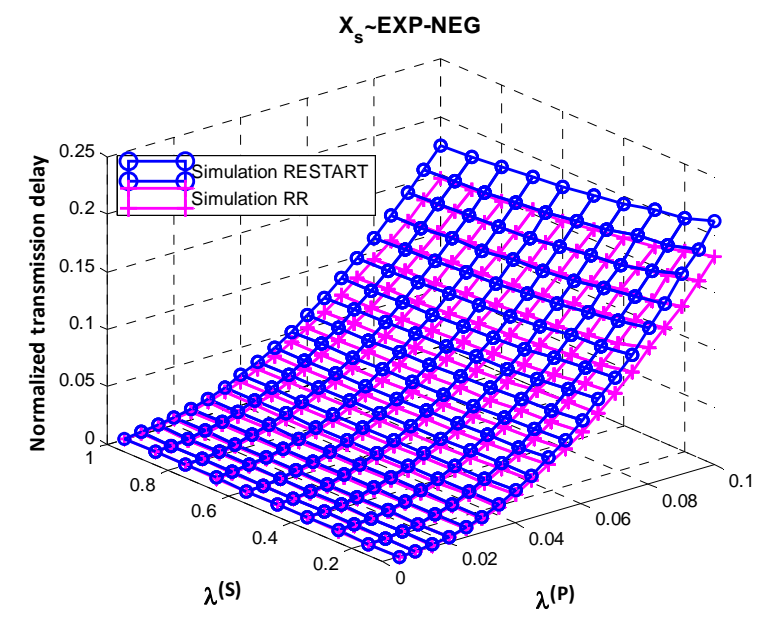

Fig. 4. Transmission delay with exponentially distributed service time and the total transmission time approximated by an exponential distribution.

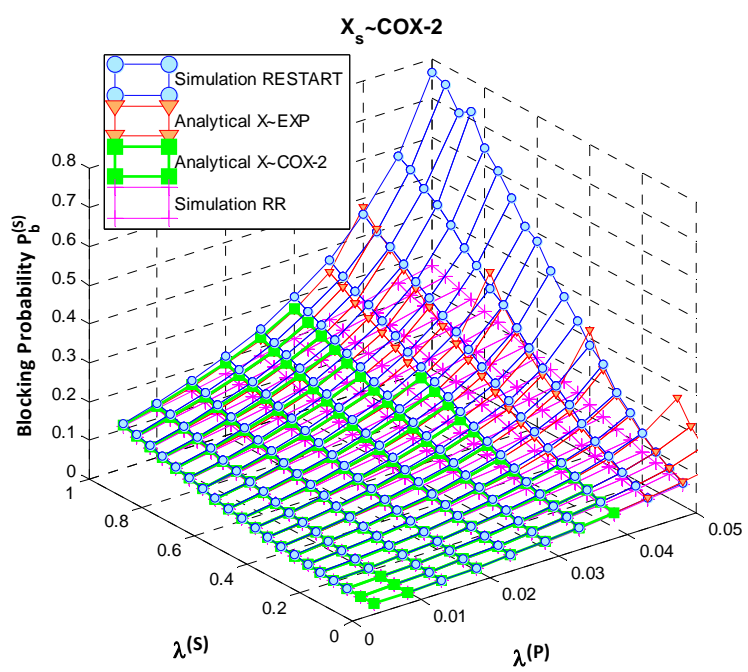

Fig. 5. Blocking probability with Coxian order 2 distributed service time and the total transmission time approximated by a $2^{\text {nd }}$ order Coxian distribution.

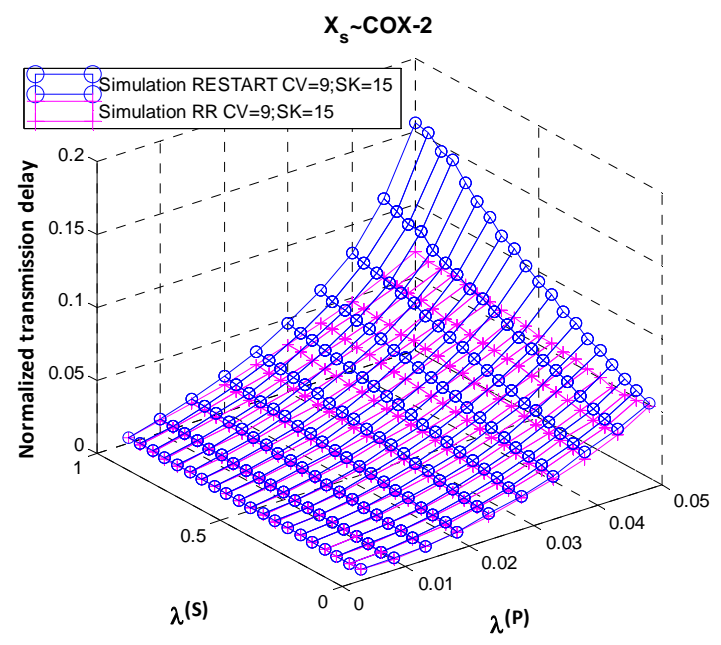

Fig. 6. Transmission delay with Coxian order 2 distributed service time and the total transmission time approximated by a $2^{\text {nd }}$ order Coxian distribution.

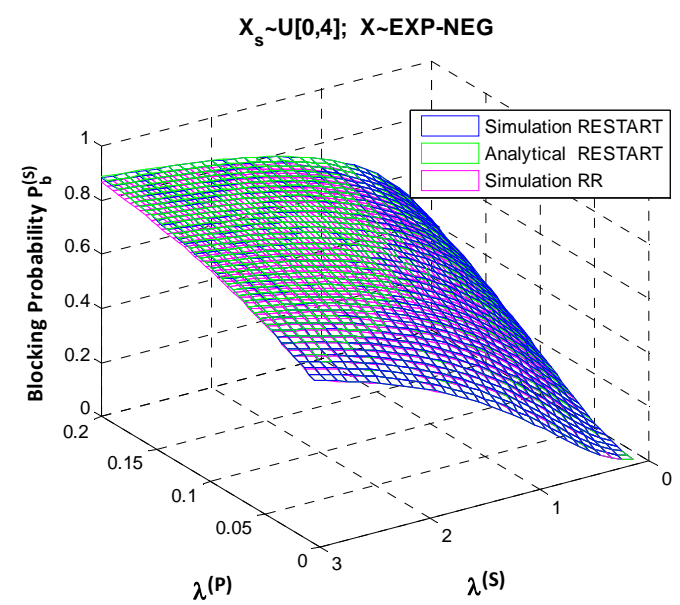

Fig. 7. Blocking probability with uniformly distributed service time and the total transmission time approximated by an exponential distribution.

Finally, Figs. 8-10 show, respectively, SU new call blocking probability, parameter $P_{I n t}$, and SU normalized transmission delay as function of both primary and secondary mean arrival rates for the case when service time for $\mathrm{SU}$ is 2-nd order Coxian distributed and channel reservation is used in the RESTART retransmission strategy. Notice that using channel reservation has the beneficial effect of reducing the negative impact of retransmissions and widens the conditions under which the system stability can be achieved. This is true for high traffic loads. This behavior can be explained as follows. Reserving resources for PU sessions prioritization over SU ones at high traffic loads decreases the effective traffic of SUs inside the system. As such, less retransmissions are needed and, therefore, the total transmission time of SUs decreases making room to accept new SU's sessions in reference with the case when no channel reservation is used. On the other hand, in [12] Fiorini et al. discussed that if the secondary unencumbered service time distribution has an exponential tail then the total transmission time distribution of SU is power tailed. They concluded that the RESTART retransmission strategy can be 
very unstable in execution of interrupted tasks. To overcome this drawback the probability $P_{\text {Int }}$ may be controlled by using a call admission control mechanism. As such, long total transmission times of SUs are reduced (fragmentation policies can be also employed to reduce the negative effects of retransmissions [18]).

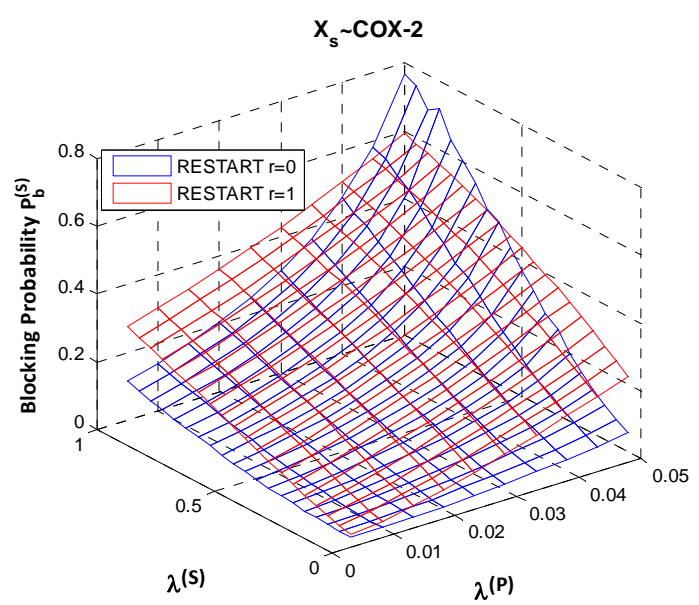

Fig. 8. Analytical results for blocking probability for RESTART strategy with channel reservation and $2^{\text {nd }}$ order Coxian service time.

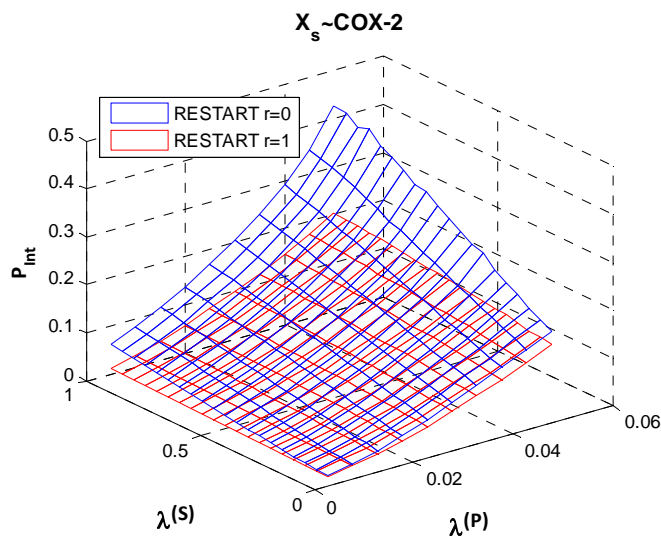

Fig. 9. Analytical results for parameter $P_{\text {Int }}$, for RESTART strategy with channel reservation and $2^{\text {nd }}$ order Coxian service time.

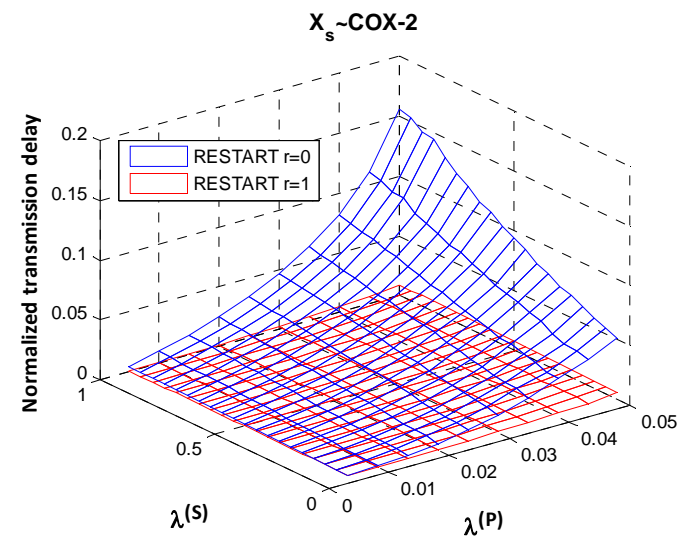

Fig. 10. Analytical results for the transmission delay for RESTART strategy with channel reservation and $2^{\text {nd }}$ order Coxian service time.

\section{REFERENCES}

[1] X. Zhu, L. Shen, and T.-S. P. Yum, "Analysis of cognitive radio spectrum access with optimal channel reservation," IEEE Commun. Lett., vol. 11, pp. 1-3, Apr. 2007.

[2] Y. Zhang, "Dynamic spectrum access in cognitive radio wireless networks," in Proc. IEEE ICC'2008, Beijing, China, May 2008, pp. 4927-4932.

[3] W. Ahmed, J. Gao, and M. Faulkner, "Performance evaluation of a cognitive radio network with exponential and truncated usage models," in Proc. IEEE ISWPC’09, Melbourne, Australia, Feb. 2009.

[4] D. Pacheco-Paramo, V. Pla, and J. Martinez-Bauset, "Optimal admission control in cognitive radio networks," IEEE CROWNCOM'09, Hannover, Germany, Jun. 2009.

[5] S. Tang and B.L. Mark, "Performance analysis of a wireless network with opportunistic spectrum sharing," in Proc. IEEE Globecom'2007, Washington, DC, USA, Nov. 2007.

[6] S. Tang and B.L. Mark, "An analytical performance model of opportunistic spectrum access in a military environment," in Proc. IEEE WCNC'2008, Las Vegas, NV, Mar.-Apr. 2008, pp. 2681-2686.

[7] S. Tang and B.L. Mark, "Analysis of opportunistic spectrum sharing with Markovian arrivals and phase-type service," IEEE Trans. Wireless Commun., vol. 8, no. 6, pp. 3142-3150, June 2009.

[8] S. Tang and B. L. Mark, "Modeling and analysis of opportunistic spectrum sharing with unreliable spectrum sensing," IEEE Trans. Wireless Commun., vol. 8, pp. 1934-1943, Apr. 2009.

[9] S. Asmussen, P. Fiorini, L.Lipsky, T., Rolski, and R. Sheahan, "Asymptotic behavior of total times for jobs that must start over if a failure occurs," Mathematics of Operations Research, vol. 33, no. 4, pp. 932-944, Nov. 2008.

[10] V. Kulkarni, V. Nicola, and K. Trivedi, "On modeling the performance and reliability of multimode systems," J. Systems Software, vol. 6, pp. 175-183, 1986.

[11] V. Kulkarni, V. Nicola, and K. Trivedi, "The completion time of a job on a multimode system," Adv. Appl. Probab., vol. 19, pp. 932-954, 1987.

[12] P. M. Fiorini, R. Sheahan, and L. Lipsky, "On unreliable computing systems when heavy-tails appear as a result of the recovery procedure," ACM SIGMETRICS Perf. Evaluation Review - Special issue on the workshop on Mathematical performance Modeling And Analysis (MAMA 2005), vol. 33, no. 2, pp.15-17, Sep. 2005.

[13] P.M. Fiorini, R. Sheahan, and L. Lipsky, "On the completion time distribution for tasks that must restart from the beginning if a failure occurs," ACM SIGMETRICS Perf. Evaluation Review, vol. 34, no. 3, Dec. 2006.

[14] P.R. Jelenkovic and J. Tan, "Can retransmissions of superexponential documents cause subexponential delays?," IEEE INFOCOM'2007, Anchorage, AK, May 2007.

[15] P.R. Jelenkovic and J. Tan, "Characterizing heavy-tailed distributions induced by retransmissions," Department of Electrical Engineering, Columbia University, New York, NY, Technical Report EE2007-09-07, Sep. 2007.

[16] J. Tan and N.B. Shroff, "Transition from heavy to light tails in retransmission durations," in Proc. IEEE INFOCOM'2010, San Diego, CA, Mar. 2010.

[17] Y.-B. Lin, S. Mohan and A. Noerpel, "Queuing priority channel assignment strategies for PCS and handoff initial access," IEEE Trans. Veh. Technol., vol. 43. no. 3, pp. 704-712, Aug. 1994.

[18] J. Nair, M. Andreasson, L.L.H. Adrew, S.H. Low, and J.C. Doyle, "File fragmentation over an unreliable channel," IEEE INFOCOM'2010, San Diego, CA, Mar. 2010.

[19] S.L. Castellanos-López, F.A. Cruz-Pérez, and G. Hernández-Valdez, "Performance of cognitive radio networks under resume and restart retransmission strategies," accepted in IEEE WiMob'2011, Shangai, China, Oct. 2011.

[20] S. L. Castellanos López, F. A. Cruz Pérez, and G. Hernández Valdez (2011). Resume and Starting-Over-Again Retransmission Strategies in Cognitive Radio Networks, Advanced Trends in Wireless Communications, Mutamed Khatib (Ed.), INTECH, available in: http://www.intechopen.com/articles/show/title/resume-and-starting-overagain-retransmission-strategies-in-cognitive-radio-networks. 